\title{
Conserving Gapless Mean-Field Theory of a Multi-Component Bose-Einstein Condensate
}

\author{
Yoshiyuki KONDO and Takafumi KITA \\ Department of Physics, Hokkaido University, Sapporo 060-0810
}

(Received April 11, 2018)

\begin{abstract}
We develop a mean-field theory for Bose-Einstein condensation of spin-1 atoms with internal degrees of freedom. It is applicable to nonuniform systems at finite temperatures with a plausible feature of satisfying the Hugenholtz-Pines theorem and various conservation laws simultaneously. Using it, we clarify thermodynamic properties and the excitation spectra of a uniform gas. The condensate is confirmed to remain in the same internal state from $T=0$ up to $T_{c}$ for both antiferromagnetic and ferromagnetic interactions. The excitation spectra of the antiferromagnetic (ferromagnetic) interaction are found to have only a single gapless mode, contrary to the prediction of the Bogoliubov theory where three (two) of them are gapless. We present a detailed discussion on those single-particle excitations in connection with the collective excitations.
\end{abstract}

KEYWORDS: Bose-Einstein condensation, mean-field theory, spinor BEC, Luttinger-Ward functional, Bogoliubov-de Gennes equation

\section{Introduction}

In 1998, Stamper-Kurn et al. ${ }^{1}$ have successfully confined ${ }^{23} \mathrm{Na}$ atoms in an optical dipole trap. They thereby realized Bose-Einstein condensation (BEC) with internal degrees of freedom corresponding to the three hyperfine spin states $\left|F=1, m_{F}= \pm 1,0\right\rangle$. Up to now, multicomponent BEC has been observed also in other systems such as ${ }^{23} \mathrm{Na}(F=2)^{2}$ and ${ }^{87} \mathrm{Rb}\left(F=1^{3,4}\right.$ and $\left.F=2^{5,6}\right)$. The internal degrees of freedom are expected to bring new physics into BEC absent in magnetically trapped single-component systems.

Several theories have been constructed for the weakly interacting Bose gases of the single-component system. The basic Bogoliubov theory ${ }^{7,8}$ is a perturbation theory without self-consistency which is applicable only near $T=0$. The Gross-Pitaevskii (GP) equation ${ }^{9-11}$ for the condensate may be regarded as the inhomogeneous Bogoliubov theory without the quasiparticle contribution. Among extensions of the Bogoliubov theory to finite temperatures is the mean-field Hartree-Fock-Bogoliubov (HFB) theory derived with the Wick decomposition procedure. However, it predicts unphysical energy gap in the excitation spectrum in contradiction to the HugenholtzPines theorem. ${ }^{12}$ To remove it, one generally introduces another approximation called the Shohno ("Popov") approximation $^{13,14}$ of neglecting the anomalous pair correlation completely. However, the approximation has a fundamental flaw of yielding dynamical equations of motion which does not satisfy various conservation laws. Thus, there are still no systematic self-consistent approximation schemes in condensed Bose systems which carry the two properties of the exact theory simultaneously: ${ }^{15}$ "gapless" and "conserving."

Theoretical studies of multi-component BEC was started by Ohmi and Machida ${ }^{16}$ and $\mathrm{Ho}^{17}$ independently in 1998. They were based on the GP equation for the spin-1 Bose gas to determine the structure of the condensate wave function at $T=0$. They have also clarified the excitation spectra by considering fluctuations around the solution of the GP equation. These pioneering works have been followed by detailed studies on the equilibrium and dynamical properties of spin-1 BEC. ${ }^{18-24}$ However, most of them consider only the region near $T=0$ based on the Bogoliubov or GP equations. In addition, other few studies at finite temperatures adopt the Shohno approximation which may not provide reliable predictions with the reasons mentioned above, especially for multicomponent systems. Thus, spin-1 BEC at finite temperatures is theoretically not well understood yet.

Recently, one of the authors has constructed a meanfield theory for a single-component BEC at finite temperatures ${ }^{14,25}$ which is both "gapless" and "conserving" with finite anomalous pair correlation. We here extend the theory to the three-component BEC. We then apply it to a uniform gas with a contact interaction to reveal its basic features as a function of temperature. As mentioned above, no detailed studies have been made at finite temperatures even for the uniform system. Especially, we wish to clarify the condensate wave function and the excitation spectra of the multi-component system at finite temperatures which may have non-trivial structures.

This paper is organized as follows. Section 2 derives a closed set of equations to determine the thermodynamic equilibrium of general nonuniform threecomponent BEC. Section 3 applies the formulation to a uniform gas under constant density. Section 4 presents numerical results for spin-dependent antiferro- and ferromagnetic interaction. Section 5 concludes the paper. In Appendix, we present the multi-component version of the Hugenholtz-Pines theorem. ${ }^{12}$ We put $\hbar=k_{\mathrm{B}}=1$ throughout. 


\section{Formulation}

\subsection{Hamiltonian}

We consider a system described by the Hamiltonian: ${ }^{16,17}$

$$
\begin{aligned}
\mathcal{H}= & \sum_{\sigma} \int \mathrm{d} \boldsymbol{r} \psi_{\sigma}^{\dagger}(\boldsymbol{r})\left(H_{0}-\mu\right) \psi_{\sigma}(\boldsymbol{r})+\frac{1}{2} \sum_{\sigma \sigma^{\prime} \tau \tau^{\prime}} \int \mathrm{d} \boldsymbol{r}_{1} \mathrm{~d} \boldsymbol{r}_{2} \\
& \times \mathcal{U}_{\sigma \sigma^{\prime}, \tau \tau^{\prime}}\left(\boldsymbol{r}_{1}-\boldsymbol{r}_{2}\right) \psi_{\sigma}^{\dagger}\left(\boldsymbol{r}_{1}\right) \psi_{\tau}^{\dagger}\left(\boldsymbol{r}_{2}\right) \psi_{\tau^{\prime}}\left(\boldsymbol{r}_{2}\right) \psi_{\sigma^{\prime}}\left(\boldsymbol{r}_{1}\right) .
\end{aligned}
$$

Here $H_{0} \equiv-\frac{\nabla^{2}}{2 M}+V_{\text {ext }}(\boldsymbol{r})$ with $M$ the mass and $V_{\text {ext }}$ the external potential, $\mu$ is the chemical potential, and $\psi_{\sigma}(\boldsymbol{r})$ is the field operator with the subscript $\sigma=0, \pm 1$ denoting the spin component. The quantity $\mathcal{U}_{\sigma \sigma^{\prime}, \tau \tau^{\prime}}$ is a spin-dependent interaction given by

$$
\begin{aligned}
& \mathcal{U}_{\sigma \sigma^{\prime}, \tau \tau^{\prime}}\left(\boldsymbol{r}_{1}-\boldsymbol{r}_{2}\right) \\
& =\delta\left(\boldsymbol{r}_{1}-\boldsymbol{r}_{2}\right)\left(c_{0} \delta_{\sigma \sigma^{\prime}} \delta_{\tau \tau^{\prime}}+c_{2} \boldsymbol{F}_{\sigma \sigma^{\prime}} \cdot \boldsymbol{F}_{\tau \tau^{\prime}}\right),
\end{aligned}
$$

where $c_{0}$ and $c_{2}$ are parameters defined in terms of spin dependent $s$-wave scattering length $a_{f}(f=0,1, \cdots)$ as $^{17}$

$$
\begin{aligned}
c_{0} & =\frac{4 \pi}{M} \frac{\left(a_{0}+2 a_{2}\right)}{3}, \\
c_{2} & =\frac{4 \pi}{M} \frac{\left(a_{2}-a_{0}\right)}{3},
\end{aligned}
$$

and $\underline{F}=\left(\boldsymbol{F}_{\sigma \sigma^{\prime}}\right)$ is the spin matrix for $F=1$ :

$$
\begin{aligned}
& \underline{F}^{1}=\frac{1}{\sqrt{2}}\left[\begin{array}{ccc}
0 & 1 & 0 \\
1 & 0 & 1 \\
0 & 1 & 0
\end{array}\right], \\
& \underline{F}^{2}=\frac{i}{\sqrt{2}}\left[\begin{array}{ccc}
0 & -1 & 0 \\
1 & 0 & -1 \\
0 & 1 & 0
\end{array}\right], \\
& \underline{F}^{3}=\left[\begin{array}{ccc}
1 & 0 & 0 \\
0 & 0 & 0 \\
0 & 0 & -1
\end{array}\right] .
\end{aligned}
$$

The second term in the round bracket of eq. (2) is similar in form with the Heisenberg spin Hamiltonian. We hence call the $c_{2}>0\left(c_{2}<0\right)$ case as antiferromagnetic (ferromagnetic).

It is convenient to introduce the Nambu vector:

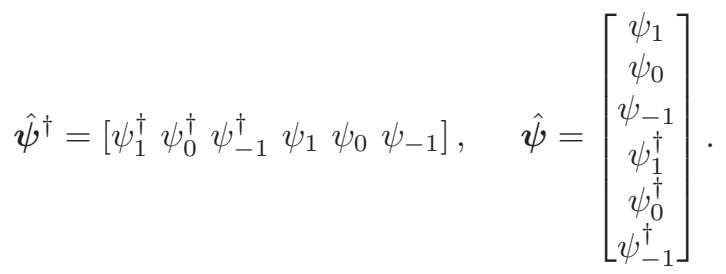

Using eq. (5) and the normal-ordering operator $\mathcal{N},{ }^{26}$ the interaction in eq. (1) is transformed into

$$
\begin{aligned}
\mathcal{H}^{\prime}=\frac{1}{8} \sum_{\nu=0}^{3} & \int \mathrm{d} \boldsymbol{r} \mathrm{d} \boldsymbol{r}^{\prime} \mathcal{U}_{\nu}\left(\boldsymbol{r}-\boldsymbol{r}^{\prime}\right) \\
& \times \mathcal{N}\left[\hat{\boldsymbol{\psi}}^{\dagger}(\boldsymbol{r}) \hat{A}^{\nu} \hat{\boldsymbol{\psi}}(\boldsymbol{r}) \hat{\boldsymbol{\psi}}^{\dagger}\left(\boldsymbol{r}^{\prime}\right) \hat{A}^{\nu} \hat{\boldsymbol{\psi}}\left(\boldsymbol{r}^{\prime}\right)\right] .
\end{aligned}
$$

Here $\mathcal{U}_{\nu}$ is defined by

$$
\mathcal{U}_{\nu}\left(\boldsymbol{r}-\boldsymbol{r}^{\prime}\right)= \begin{cases}c_{0} \delta\left(\boldsymbol{r}-\boldsymbol{r}^{\prime}\right) & : \nu=0 \\ c_{2} \delta\left(\boldsymbol{r}-\boldsymbol{r}^{\prime}\right) & : \nu=1,2,3\end{cases}
$$

and $\hat{A}^{\nu}$ denotes the vertex:

$$
\hat{A}^{\nu}=\left[\begin{array}{ll}
\underline{A}^{\nu} & \underline{0}^{\nu} \\
\underline{A} & \left(\underline{A}^{\mathrm{T}}\right.
\end{array}\right],
$$

with $\underline{A}^{\nu}$ defined in terms of the unit matrix $\underline{1}$ and eq. (4) as

$$
\underline{A}^{\nu}=\left\{\begin{array}{ll}
\underline{1} & : \nu=0 \\
\underline{F}^{\nu} & : \nu=1,2,3
\end{array} .\right.
$$

The equivalence between the interaction term in eq. (1) and eq. (6) can be checked easily by writing the latter without using $\mathcal{N}$. The expression (6) has an advantage that the perturbation expansion with respect to $\mathcal{H}^{\prime}$ can be carried out directly by using the Nambu Green's function; see Appendix A of ref. 14 for details.

\subsection{Mean-field equations}

To describe the condensed phase, let us express the field operator $\boldsymbol{\psi}(\boldsymbol{r}) \equiv\left[\psi_{1}(\boldsymbol{r}) \psi_{0}(\boldsymbol{r}) \psi_{-1}(\boldsymbol{r})\right]^{\mathrm{T}}$ as a sum of the condensate wave function $\boldsymbol{\Psi}(\boldsymbol{r})$ and the quasiparticle field $\phi(\boldsymbol{r})$ as

$$
\boldsymbol{\psi}(\boldsymbol{r})=\boldsymbol{\Psi}(\boldsymbol{r})+\phi(\boldsymbol{r}) .
$$

We next introduce the Matsubara Green's function in the Nambu space as

$$
\begin{aligned}
\hat{G}\left(\boldsymbol{r}, \boldsymbol{r}^{\prime}, \tau\right) & =-\hat{\tau}_{3}\left\langle T_{\tau} \hat{\boldsymbol{\phi}}(\boldsymbol{r}, \tau) \hat{\phi}^{\dagger}\left(\boldsymbol{r}^{\prime}\right)\right\rangle \\
& \equiv\left[\begin{array}{cc}
\underline{G}\left(\boldsymbol{r}, \boldsymbol{r}^{\prime}, \tau\right) & \underline{F}\left(\boldsymbol{r}, \boldsymbol{r}^{\prime}, \tau\right) \\
-\underline{F}^{*}\left(\boldsymbol{r}, \boldsymbol{r}^{\prime}, \tau\right) & -\underline{G}^{*}\left(\boldsymbol{r}, \boldsymbol{r}^{\prime}, \tau\right)
\end{array}\right],
\end{aligned}
$$

with $\hat{\tau}_{3}$ denoting the third Pauli matrix in the Nambu space. Equation (11b) can be Fourier-transformed as

$$
\hat{G}\left(\boldsymbol{r}, \boldsymbol{r}^{\prime}, \tau\right)=\frac{1}{\beta} \sum_{n} \hat{G}\left(\boldsymbol{r}, \boldsymbol{r}^{\prime}, i \omega_{n}\right) e^{-i \omega_{n} \tau},
$$

where $\beta \equiv T^{-1}$ and $\omega_{n}$ is the Matsubara frequency $\omega_{n}=$ $2 n \pi / \beta$ with $n=0, \pm 1, \pm 2, \cdots$.

Using eq. (12), we now write down our mean-field Luttinger-Ward functional for BEC as ${ }^{14,27}$

$$
\begin{aligned}
& \Omega=\int \mathrm{d} \boldsymbol{r} \Psi^{\dagger}(\boldsymbol{r})\left(H_{0}-\mu\right) \Psi(\boldsymbol{r}) \\
& +\frac{1}{2 \beta} \sum_{n=-\infty}^{\infty} \operatorname{Tr}\left[\ln \left(\hat{K}+\hat{\Sigma}-i \omega_{n} \hat{1}\right)+\hat{G} \hat{\Sigma}\right] \hat{1}\left(\omega_{n}\right)+\Phi .
\end{aligned}
$$

Here $\hat{K}$ is defined by $\hat{K} \equiv \hat{\tau}_{3}\left(H_{0}-\mu\right), \hat{1}\left(i \omega_{n}\right)$ denotes

$$
\hat{1}\left(\omega_{n}\right)=\left[\begin{array}{cc}
\underline{1}^{i \omega_{n} 0^{+}} & \underline{0} \\
\underline{0} & \underline{1} \mathrm{e}^{-i \omega_{n} 0^{+}}
\end{array}\right],
$$

with $0^{+}$an infinitesimal positive constant, and $\operatorname{Tr}$ includes integration over space variables. The quantity $\hat{\Sigma}$ is the proper self-energy obtained from the functional $\Phi=\Phi\left[\Psi, \Psi^{*}, \hat{G}\right]$ by

$$
\hat{\Sigma}\left(\boldsymbol{r}, \boldsymbol{r}^{\prime}, \omega_{n}\right)=-2 \beta \frac{\delta \Phi}{\delta \hat{G}\left(\boldsymbol{r}^{\prime}, \boldsymbol{r}, \omega_{n}\right)} .
$$

With eq. (15), functional $\Omega$ is stationary with respect to a variation of $\hat{G}$ which satisfies Dyson's equation:

$$
\hat{G}^{-1}=i \omega_{n} \hat{1}-\hat{K}-\hat{\Sigma} .
$$


The condensate wave function $\boldsymbol{\Psi}$ in equilibrium is also determined by $\delta \Omega / \delta \boldsymbol{\Psi}^{*}=0$. Noting $\delta \Omega / \delta \hat{G}=0$, we obtain

$$
\left(H_{0}-\mu\right) \Psi(\boldsymbol{r})+\frac{\delta \Phi}{\delta \Psi^{*}(\boldsymbol{r})}=0 .
$$

Equations (15) and (17) constitute the $\Phi$-derivative approximation where various conservation laws are obeyed automatically. This is one of the main advantages of using the Luttinger-Ward functional.

Now, we choose $\Phi$ so that the Hugenholtz-Pines theorem is satisfied simultaneously; see Appendix for the multi-component version of the Hugenholtz-Pines theorem. Explicitly, our $\Phi$ is given by

$$
\begin{aligned}
& \Phi=\frac{1}{2} \sum_{\nu} \int \mathrm{d} \boldsymbol{r} \mathrm{d} \boldsymbol{r}^{\prime} \mathcal{U}_{\nu}\left(\boldsymbol{r}-\boldsymbol{r}^{\prime}\right) \\
& \times\left\{\frac{1}{2} \operatorname{Tr}\left[\hat{A}^{\nu} \hat{\mathbf{\Psi}}(\boldsymbol{r}) \hat{\Psi}^{\dagger}(\boldsymbol{r})\right] \frac{1}{2} \operatorname{Tr}\left[\hat{A}^{\nu} \hat{\mathbf{\Psi}}\left(\boldsymbol{r}^{\prime}\right) \hat{\mathbf{\Psi}}^{\dagger}\left(\boldsymbol{r}^{\prime}\right)\right]\right. \\
& -\frac{2}{\beta} \sum_{n}\left(\frac{1}{2} \operatorname{Tr}\left[\hat{A}^{\nu} \hat{\mathbf{\Psi}}(\boldsymbol{r}) \hat{\Psi}^{\dagger}(\boldsymbol{r})\right]\right. \\
& \quad \times \frac{1}{2} \operatorname{Tr}\left[\hat{A}^{\nu} \hat{\tau}_{3} \hat{G}\left(\boldsymbol{r}^{\prime}, \boldsymbol{r}^{\prime}, i \omega_{n}\right) \hat{1}\left(\omega_{n}\right)\right] \\
& \left.+\frac{1}{2} \operatorname{Tr}\left[\hat{A}^{\nu} \hat{\tau}_{3} \hat{\boldsymbol{\Psi}}(\boldsymbol{r}) \hat{\mathbf{\Psi}}^{\dagger}\left(\boldsymbol{r}^{\prime}\right) \hat{A}^{\nu} \hat{G}\left(\boldsymbol{r}^{\prime}, \boldsymbol{r}, \omega_{n}\right) \hat{1}\left(\omega_{n}\right)\right]\right) \\
& +\frac{1}{\beta^{2}} \sum_{n, m}\left(\frac{1}{2} \operatorname{Tr}\left[\hat{A}^{\nu} \hat{\tau}_{3} \hat{G}\left(\boldsymbol{r}, \boldsymbol{r}, \omega_{n}\right)\right]\right. \\
& \quad \times \frac{1}{2} \operatorname{Tr}\left[\hat{A}^{\nu} \hat{\tau}_{3} \hat{G}\left(\boldsymbol{r}^{\prime}, \boldsymbol{r}^{\prime}, \omega_{m}\right)\right] \\
& \left.\left.+\frac{1}{2} \operatorname{Tr}\left[\hat{A}^{\nu} \hat{G}\left(\boldsymbol{r}, \boldsymbol{r}^{\prime}, \omega_{n}\right) \hat{1}\left(\omega_{n}\right) \hat{A}^{\nu} \hat{G}\left(\boldsymbol{r}^{\prime}, \boldsymbol{r}, \omega_{m}\right) \hat{1}\left(\omega_{m}\right)\right]\right)\right\} .
\end{aligned}
$$

This functional $\Phi$ is different from $\Phi_{\mathrm{HFB}}$ of the HFB theory in the Fock terms, i.e., the third and the fifth terms on the right-hand side of eq. (18). Indeed, $\Phi_{\mathrm{HFB}}$ is recovered from $\Phi$ by replacing $\hat{\tau}_{3} \hat{\Psi} \hat{\Psi}^{\dagger} \rightarrow \hat{\Psi} \hat{\Psi}^{\dagger}$ and $\hat{G} \rightarrow \hat{\tau}_{3} \hat{G}$. Put it another way, $\Phi$ is obtained from $\Phi_{\mathrm{HFB}}$ by the following subtraction:

$$
\begin{aligned}
& \Phi= \Phi_{\mathrm{HFB}}-\sum_{\nu} \int \mathrm{d} \boldsymbol{r} \mathrm{d} \boldsymbol{r}^{\prime} \mathcal{U}_{\nu}\left(\boldsymbol{r}-\boldsymbol{r}^{\prime}\right) \\
& \times\left(-\frac{1}{\beta} \sum_{n} \operatorname{Tr}\left[\hat{A}^{\nu} \hat{\mathbf{\Psi}}(\boldsymbol{r}) \hat{\Psi}^{\dagger}\left(\boldsymbol{r}^{\prime}\right) \hat{A}^{\nu} \hat{\tau}_{3} \hat{F}\left(\boldsymbol{r}^{\prime}, \boldsymbol{r}, \omega_{n}\right) \hat{1}\left(\omega_{n}\right)\right]\right. \\
&+\frac{1}{2 \beta^{2}} \sum_{n, m} \operatorname{Tr}\left[\hat{A}^{\nu} \hat{\tau}_{3} \hat{G}\left(\boldsymbol{r}, \boldsymbol{r}^{\prime}, \omega_{n}\right) \hat{1}\left(\omega_{n}\right)\right. \\
&\left.\left.\times \hat{A}^{\nu} \hat{\tau}_{3} \hat{F}\left(\boldsymbol{r}^{\prime}, \boldsymbol{r}, \omega_{m}\right) \hat{1}\left(\omega_{m}\right)\right]\right)
\end{aligned}
$$

with

$$
\hat{F}\left(\boldsymbol{r}, \boldsymbol{r}^{\prime}, \omega_{n}\right)=\left[\begin{array}{cc}
\underline{0} & \underline{F}\left(\boldsymbol{r}, \boldsymbol{r}^{\prime}, \omega_{n}\right) \\
-\underline{F^{*}}\left(\boldsymbol{r}, \boldsymbol{r}^{\prime}, \omega_{n}\right) & \underline{0}
\end{array}\right] .
$$

This subtraction has the effect to cancel the overcounting of the anomalous pair correlation in the HFB theory, thereby leading to a mean-field theory which satisfies the Hugenholtz-Pines theorem.
Now, the proper self-energy is obtained with eqs. (15) and (18) as

$$
\begin{aligned}
& \hat{\Sigma}\left(\boldsymbol{r}, \boldsymbol{r}^{\prime}\right)=\left[\begin{array}{cc}
\underline{\Sigma}\left(\boldsymbol{r}, \boldsymbol{r}^{\prime}\right) & \underline{\Delta}\left(\boldsymbol{r}, \boldsymbol{r}^{\prime}\right) \\
-\underline{\Delta}^{*}\left(\boldsymbol{r}, \boldsymbol{r}^{\prime}\right) & -\underline{\Sigma}^{*}\left(\boldsymbol{r}, \boldsymbol{r}^{\prime}\right)
\end{array}\right] \\
& =\sum_{\nu}\left\{\frac{1}{2} \delta\left(\boldsymbol{r}-\boldsymbol{r}^{\prime}\right) \hat{A}^{\nu} \hat{\tau}_{3} \int \mathrm{d} \boldsymbol{r}^{\prime \prime} \mathcal{U}_{\nu}\left(\boldsymbol{r}-\boldsymbol{r}^{\prime \prime}\right)\right. \\
& \left.\quad \times \operatorname{Tr}\left[\hat{A}^{\nu} \hat{\tau}_{3} \hat{\rho}\left(\boldsymbol{r}^{\prime \prime}, \boldsymbol{r}^{\prime \prime}\right)\right]+\mathcal{U}_{\nu}\left(\boldsymbol{r}-\boldsymbol{r}^{\prime}\right) \hat{A}^{\nu} \hat{\rho}\left(\boldsymbol{r}, \boldsymbol{r}^{\prime}\right) \hat{A}^{\nu}\right\},
\end{aligned}
$$

where $\hat{\rho}$ is the one-particle density matrix in the Nambu space defined by

$$
\begin{aligned}
& \hat{\rho}\left(\boldsymbol{r}, \boldsymbol{r}^{\prime}\right)=\left[\begin{array}{cc}
\underline{\rho}\left(\boldsymbol{r}, \boldsymbol{r}^{\prime}\right) & \underline{\tilde{\rho}}\left(\boldsymbol{r}, \boldsymbol{r}^{\prime}\right) \\
-\underline{\tilde{\rho}}^{*}\left(\boldsymbol{r}, \boldsymbol{r}^{\prime}\right) & -\underline{\rho}^{*}\left(\boldsymbol{r}, \boldsymbol{r}^{\prime}\right)
\end{array}\right] \\
& =\hat{\tau}_{3} \vec{\Psi}(\boldsymbol{r}) \vec{\Psi}^{\dagger}\left(\boldsymbol{r}^{\prime}\right)+\hat{\rho}^{(\mathrm{qp})}\left(\boldsymbol{r}, \boldsymbol{r}^{\prime}\right),
\end{aligned}
$$

with

$$
\hat{\rho}^{(\mathrm{qp})}\left(\boldsymbol{r}, \boldsymbol{r}^{\prime}\right) \equiv-\frac{1}{\beta} \sum_{n} \hat{G}\left(\boldsymbol{r}, \boldsymbol{r}^{\prime}, \omega_{n}\right) \hat{1}\left(\omega_{n}\right) .
$$

The submatrices $\underline{\Sigma}$ and $\underline{\Delta}$ satisfy $\underline{\Sigma}^{*}\left(\boldsymbol{r}, \boldsymbol{r}^{\prime}\right)=\underline{\Sigma}^{\mathrm{T}}\left(\boldsymbol{r}^{\prime}, \boldsymbol{r}\right)$ and $\underline{\Delta}\left(\boldsymbol{r}, \boldsymbol{r}^{\prime}\right)=\underline{\Delta}^{\mathrm{T}}\left(\boldsymbol{r}^{\prime}, \boldsymbol{r}\right)$. Similarly, we have $\underline{\rho}^{*}\left(\boldsymbol{r}, \boldsymbol{r}^{\prime}\right)=$ $\underline{\rho}^{\mathrm{T}}\left(\boldsymbol{r}^{\prime}, \boldsymbol{r}\right)$ and $\underline{\tilde{\rho}}^{\mathrm{T}}\left(\boldsymbol{r}, \boldsymbol{r}^{\prime}\right)=\underline{\tilde{\rho}}\left(\boldsymbol{r}^{\prime}, \boldsymbol{r}\right)$.

Next, eqs. (17) and (18) lead to the the equation for the condensate wave function:

$$
\begin{aligned}
& \left(H_{0}-\mu\right) \boldsymbol{\Psi}(\boldsymbol{r}) \\
& \quad+\int \mathrm{d} \boldsymbol{r}^{\prime}\left[\underline{\Sigma}\left(\boldsymbol{r}, \boldsymbol{r}^{\prime}\right) \boldsymbol{\Psi}(\boldsymbol{r})-\underline{\Delta}\left(\boldsymbol{r}, \boldsymbol{r}^{\prime}\right) \boldsymbol{\Psi}^{*}\left(\boldsymbol{r}^{\prime}\right)\right]=\underline{0} .
\end{aligned}
$$

Equation (24) is the GP equation in our mean-field theory. In the homogeneous case, we can write $\boldsymbol{\Psi} \rightarrow \sqrt{n_{0}} \boldsymbol{\eta}$, where $n_{0}$ is the condensate density and $\boldsymbol{\eta}(|\boldsymbol{\eta}|=1)$ specifies the direction of the order parameter. Equation (24) then reduces to $\mu \boldsymbol{\zeta}=\underline{\Sigma}_{\boldsymbol{k}=0} \boldsymbol{\zeta}-\underline{\Delta}_{\boldsymbol{k}=0} \boldsymbol{\zeta}^{*}$, which is the Hugenholtz-Pines relation of the multi-component case; see eq. (A.9) in Appendix. It tells us the existence of a single gapless excitation spectrum in the system, as shown explicitly below eq. (39). Note that $\mu$ and $\boldsymbol{\eta}$ should be determined here as the lowest eigenvalue and its eigenstate, respectively. Finally, the thermodynamic relation $N=-\partial \Omega / \partial \mu$ yields the expression for the total particle number as $N=\operatorname{Tr} \int \underline{\rho}(\boldsymbol{r}, \boldsymbol{r}) \mathrm{d} \boldsymbol{r}$.

In order to diagonalize Green's function which satisfies eq. (16), we consider the Bogoliubov-de Gennes (BdG) equation:

$$
\int \mathrm{d} \boldsymbol{r}^{\prime} \hat{H}\left(\boldsymbol{r}, \boldsymbol{r}^{\prime}\right)\left[\begin{array}{c}
\boldsymbol{u}_{j}\left(\boldsymbol{r}^{\prime}\right) \\
-\boldsymbol{v}_{j}^{*}\left(\boldsymbol{r}^{\prime}\right)
\end{array}\right]=E_{j}\left[\begin{array}{c}
\boldsymbol{u}_{j}(\boldsymbol{r}) \\
-\boldsymbol{v}_{j}^{*}(\boldsymbol{r})
\end{array}\right],
$$

where $\hat{H}\left(\boldsymbol{r}, \boldsymbol{r}^{\prime}\right) \equiv \hat{K}(\boldsymbol{r}) \delta\left(\boldsymbol{r}-\boldsymbol{r}^{\prime}\right)+\hat{\Sigma}\left(\boldsymbol{r}, \boldsymbol{r}^{\prime}\right)$. We assume that the eigenstate for $E_{j}>0$ can be normalized as

$$
\left\langle\boldsymbol{u}_{j} \mid \boldsymbol{u}_{j^{\prime}}\right\rangle-\left\langle\boldsymbol{v}_{j} \mid \boldsymbol{v}_{j^{\prime}}\right\rangle^{*}=\delta_{j j^{\prime}} .
$$

It then follows from the symmetry of $\hat{H}$ that the eigenvector with the eigenvalue $-E_{j}$ is given by $\left[-\boldsymbol{v}_{j}^{\mathrm{T}}, \boldsymbol{u}_{j}^{\dagger}\right]^{\mathrm{T}}{ }^{14}$ Let us introduce matrices $\hat{U}$ and $\hat{E}$ by

$$
\hat{U} \equiv\left[\begin{array}{cc}
\underline{U} & -\underline{V} \\
-\underline{V}^{*} & \underline{U^{*}}
\end{array}\right], \quad \hat{E} \equiv\left[\begin{array}{cc}
\underline{E} & \underline{0} \\
\underline{0} & -\underline{E}
\end{array}\right],
$$


with $\underline{U} \equiv\left(\boldsymbol{u}_{j_{1}} \boldsymbol{u}_{j_{2}} \cdots\right), \underline{V} \equiv\left(\boldsymbol{v}_{j_{1}} \boldsymbol{v}_{j_{2}} \cdots\right)$ and $(\underline{E})_{j j^{\prime}}=$ $E_{j} \delta_{j j^{\prime}}$. Using these matrices, the BdG equation can be written compactly as $\int \mathrm{d} \boldsymbol{r}^{\prime} \hat{H}\left(\boldsymbol{r}, \boldsymbol{r}^{\prime}\right) \hat{U}\left(\boldsymbol{r}^{\prime}\right)=\hat{U}(\boldsymbol{r}) \hat{E}$. It hence follows that we can expand Green's function as

$$
\hat{G}\left(\boldsymbol{r}, \boldsymbol{r}^{\prime}, \omega_{n}\right)=\hat{U}(\boldsymbol{r})\left(i \omega_{n} \hat{1}-\hat{E}\right)^{-1} \hat{U}^{\dagger}\left(\boldsymbol{r}^{\prime}\right) .
$$

We now carry out the summation over the Matsubara frequency in eq. (23). We then obtain the quasiparticle contribution to the density matrix in eq. (22) as

$$
\begin{gathered}
\underline{\rho}^{(\mathrm{qp})}\left(\boldsymbol{r}, \boldsymbol{r}^{\prime}\right)=\left\langle T_{\tau} \phi\left(\boldsymbol{r}, 0^{-}\right) \phi^{\dagger}\left(\boldsymbol{r}^{\prime}\right)\right\rangle \\
=\left\{\underline{U}(\boldsymbol{r}) f(\underline{E}) \underline{U}^{\dagger}\left(\boldsymbol{r}^{\prime}\right)+\underline{V}(\boldsymbol{r})[\underline{1}+f(\underline{E})] \underline{V}^{\dagger}\left(\boldsymbol{r}^{\prime}\right)\right\}, \\
\underline{\tilde{\rho}}^{(\mathrm{qp})}\left(\boldsymbol{r}, \boldsymbol{r}^{\prime}\right)=\left\langle\phi(\boldsymbol{r}) \phi\left(\boldsymbol{r}^{\prime}\right)\right\rangle \\
=\frac{1}{2}\left\{\underline{V}(\boldsymbol{r})[\underline{1}+2 f(\underline{E})] \underline{U}^{\mathrm{T}}\left(\boldsymbol{r}^{\prime}\right)\right. \\
\left.+\underline{U}(\boldsymbol{r})[\underline{1}+2 f(\underline{E})] \underline{V}^{\mathrm{T}}\left(\boldsymbol{r}^{\prime}\right)\right\},
\end{gathered}
$$

where $f$ is the Bose distribution function.

Equations (24) and (25) with eqs. (21), (22) and (29) form a closed set of self-consistent equations to determine the equilibrium.

Using eqs. (24) and (25), we can transform the thermodynamic potential of eq. (13) into

$$
\begin{gathered}
\Omega_{\mathrm{eq}}=\beta^{-1} \sum_{j} \ln \left(1-\mathrm{e}^{-\beta E_{j}}\right)-\sum_{j} E_{j} \int\left|\boldsymbol{v}_{j}(\boldsymbol{r})\right|^{2} \mathrm{~d} \boldsymbol{r} \\
-\frac{1}{2} \int \mathrm{d} \boldsymbol{r} \mathrm{d} \boldsymbol{r}^{\prime} \operatorname{Tr}\left[\underline{\Sigma}\left(\boldsymbol{r}, \boldsymbol{r}^{\prime}\right) \underline{\rho}\left(\boldsymbol{r}^{\prime}, \boldsymbol{r}\right)-\underline{\Delta}\left(\boldsymbol{r}, \boldsymbol{r}^{\prime}\right) \underline{\tilde{\rho}}\left(\boldsymbol{r}^{\prime}, \boldsymbol{r}\right)\right] .
\end{gathered}
$$

Also, entropy is obtained as

$$
S=-\sum_{j}\left\{f\left(E_{j}\right) \ln f\left(E_{j}\right)-\left[1+f\left(E_{j}\right)\right] \ln \left[1+f\left(E_{j}\right)\right]\right\} .
$$

Finally, the specific heat is calculated by the thermodynamic relation $C=-T \partial S / \partial T$.

\section{Uniform Gas under Constant Density}

\subsection{Equations to determine equilibrium}

We now apply the previous formulation to a uniform Bose gas with fixed density $n \equiv N / \mathcal{V}$, where $\mathcal{V}$ denotes volume of the system. In this case, the condensate wave function $\boldsymbol{\Psi}$ can be written as

$$
\boldsymbol{\Psi}=\sqrt{n_{0}} \boldsymbol{\eta},
$$

with $n_{0} \equiv N_{0} / \mathcal{V}$ the condensate density and $\boldsymbol{\eta}$ a constant vector with $|\boldsymbol{\eta}|=1$. Also, eq. (22) may be expanded in plane waves as

$$
\hat{\rho}\left(\boldsymbol{r}, \boldsymbol{r}^{\prime}\right)=\frac{1}{\mathcal{V}} \sum_{\boldsymbol{k}}\left[\begin{array}{cc}
\underline{\rho}(\boldsymbol{k}) & \underline{\tilde{\rho}}(\boldsymbol{k}) \\
-\underline{\tilde{\rho}}(-\boldsymbol{k}) & -\underline{\rho}(-\boldsymbol{k})
\end{array}\right] \mathrm{e}^{i \boldsymbol{k} \cdot\left(\boldsymbol{r}-\boldsymbol{r}^{\prime}\right)},
$$

with

$$
\begin{aligned}
& \underline{\rho}(\boldsymbol{k})=\delta_{\boldsymbol{k} \mathbf{0}} N_{0} \boldsymbol{\eta} \boldsymbol{\eta}^{\dagger}+\underline{\rho}^{(\mathrm{qp})}(\boldsymbol{k}), \\
& \underline{\tilde{\rho}}(\boldsymbol{k})=\delta_{\boldsymbol{k} \mathbf{0}} N_{0} \boldsymbol{\eta} \boldsymbol{\eta}^{\mathrm{T}}+\underline{\tilde{\rho}}^{(\mathrm{qp})}(\boldsymbol{k}) .
\end{aligned}
$$

The transition temperature of the noninteracting threecomponent $\mathrm{BEC}$ is given by

$$
T_{0}=\frac{2 \pi}{M}\left[\frac{n}{3 \zeta\left(\frac{3}{2}\right)}\right]^{\frac{2}{3}}
$$

where $\zeta\left(\frac{3}{2}\right) \simeq 2.612$ is the Riemann $\zeta$-function. This $T_{0}$ is $3^{-2 / 3}$ times the transition temperature of the onecomponent BEC.

The self-energy (21) becomes $\boldsymbol{k}$-independent in momentum space for the contact interaction of eq. (7). Indeed, $\underline{\Sigma}$ and $\underline{\Delta}$ in $\boldsymbol{k}$ space are obtained as

$$
\frac{\underline{\underline{\Sigma}}}{T_{0}}=\frac{2}{N}\left[3 \zeta\left(\frac{3}{2}\right)\right]^{2 / 3} \sum_{\nu \boldsymbol{k}} \delta_{\nu}\left[\underline{A}^{\nu} \operatorname{Tr} \underline{\rho}(\boldsymbol{k}) \underline{A}^{\nu}+\underline{A}^{\nu} \underline{\rho}(\boldsymbol{k}) \underline{A}^{\nu}\right],
$$

$$
\frac{\underline{\Delta}}{T_{0}}=\frac{2}{N}\left[3 \zeta\left(\frac{3}{2}\right)\right]^{2 / 3} \sum_{\nu \boldsymbol{k}} \delta_{\nu} \underline{A}^{\nu} \underline{\tilde{\rho}}(\boldsymbol{k})\left(\underline{A}^{\nu}\right)^{\mathrm{T}},
$$

where $\delta_{\nu}$ denotes dimensionless interaction strength defined in terms of eq. (3) by

$$
\delta_{\nu}=\left\{\begin{array}{ll}
\frac{M}{4 \pi} c_{0} n^{1 / 3} & : \nu=0 \\
\frac{M}{4 \pi} c_{2} n^{1 / 3} & : \nu=1,2,3
\end{array} .\right.
$$

The GP equation (24) is transformed by using eq. (32) into

$$
\mu \boldsymbol{\eta}=\underline{\Sigma} \boldsymbol{\eta}-\underline{\Delta} \boldsymbol{\eta}^{*} .
$$

This is the Hugenholtz-Pines relation of the threecomponent system; see Appendix for details. The BdG equation (25) now reads

$$
\hat{H}_{\boldsymbol{k}}\left[\begin{array}{c}
\boldsymbol{u}_{\boldsymbol{k} \sigma} \\
-\boldsymbol{v}_{\boldsymbol{k} \sigma}^{*}
\end{array}\right]=E_{\boldsymbol{k} \sigma}\left[\begin{array}{c}
\boldsymbol{u}_{\boldsymbol{k} \sigma} \\
-\boldsymbol{v}_{\boldsymbol{k} \sigma}^{*}
\end{array}\right],
$$

where $\hat{H}_{\boldsymbol{k}} \equiv \hat{\tau}_{3}\left(\frac{k^{2}}{2 M}-\mu\right)+\hat{\Sigma}$, the eigenstate is normalized as $\left|u_{\boldsymbol{k} \sigma}\right|^{2}-\left|v_{\boldsymbol{k} \sigma}\right|^{2}=1$, and the subscript $\sigma=0, \pm 1$ distinguishes the three eigenvalues of each $\boldsymbol{k}$. This eigenvalue problem includes eq. (38) as the special case for $E_{\boldsymbol{k}=\mathbf{0} \sigma}=0$ with $u_{\boldsymbol{k}=\mathbf{0} \sigma}=v_{\boldsymbol{k}=\mathbf{0} \sigma}=\boldsymbol{\zeta}$. It hence follows that the quasiparticle excitation is gapless as $\boldsymbol{k} \rightarrow \mathbf{0}$ at least for one branch among $\sigma=0, \pm 1$. The quasiparticle contribution (29) to the density matrix is now given by

$\underline{\rho}^{(\mathrm{qp})}(\boldsymbol{k})=\left\{\underline{U}(\boldsymbol{k}) f\left(\underline{E}_{k}\right) \underline{U}^{\dagger}(\boldsymbol{k})+\underline{V}(\boldsymbol{k})\left[\underline{1}+f\left(\underline{E}_{\boldsymbol{k}}\right)\right] \underline{V}^{\dagger}(\boldsymbol{k})\right\}$,

$$
\begin{aligned}
\underline{\tilde{\rho}}^{(\mathrm{qp})}(\boldsymbol{k})= & \frac{1}{2}\left\{\underline{V}(\boldsymbol{k})\left[\underline{1}+2 f\left(\underline{E}_{\boldsymbol{k}}\right)\right] \underline{U}^{\mathrm{T}}(\boldsymbol{k})\right. \\
& \left.+\underline{U}(\boldsymbol{k})\left[\underline{1}+2 f\left(\underline{E}_{\boldsymbol{k}}\right)\right] \underline{V}^{\mathrm{T}}(\boldsymbol{k})\right\},
\end{aligned}
$$

with $\underline{U}(\boldsymbol{k}) \equiv\left[\begin{array}{lll}\boldsymbol{u}_{\boldsymbol{k} 1} & \boldsymbol{u}_{\boldsymbol{k} 0} & \boldsymbol{u}_{\boldsymbol{k}-1}\end{array}\right]$ and $\underline{V}(\boldsymbol{k}) \equiv\left[\begin{array}{lll}\boldsymbol{v}_{\boldsymbol{k} 1} & \boldsymbol{v}_{\boldsymbol{k} 0} & \boldsymbol{v}_{\boldsymbol{k}-1}\end{array}\right]$. Finally, we sum eq. (34a) over $\boldsymbol{k}$ and take trace of it subsequently. We thereby obtain an expression for the condensate density as

$$
\frac{n_{0}}{n}=1-\frac{1}{N} \sum_{\boldsymbol{k}} \operatorname{Tr} \underline{\rho}^{(\mathrm{qp})}(\boldsymbol{k}) .
$$




\subsection{Numerical procedures}

Numerical calculations of the above self-consistent equations have been carried out as follows.

(i) The chemical potential $\mu$ and the vector $\boldsymbol{\eta}$ are determined by eq. (38) as the smallest eigenvalue and its eigenstate. To carry this out, we have to know the expressions of $n_{0}, \underline{\rho}^{(\mathrm{qp})}(\boldsymbol{k})$ and $\tilde{\rho}^{(\mathrm{qp})}(\boldsymbol{k})$ in advance, as seen from eqs. (34) and (36). We use the values from the previous calculation for them; we initially set $n_{0}=n$ and $\underline{\rho}^{(\mathrm{qp})}=\underline{\tilde{\rho}}^{(\mathrm{qp})}=\underline{0}$ to start the whole calculation at $T=0$. $\bar{W}$ e determine $\boldsymbol{\eta}$ self-consistently so that it actually corresponds to the eigenstate of the smallest eigenvalue.

(ii) Using $\mu$ and $\hat{\Sigma}$ thus calculated, we solve the BdG equation (39) to obtain the quasi-particle spectrum $E_{\boldsymbol{k} \sigma}$ and the matrix $\hat{U}_{\boldsymbol{k}}$.

(iii) With $E_{\boldsymbol{k} \sigma}$ and $\hat{U}_{\boldsymbol{k}}$, we compute $\rho^{(\mathrm{qp})}(\boldsymbol{k}), \tilde{\rho}^{(\mathrm{qp})}(\boldsymbol{k})$ and $n_{0}$ by eqs. (40a), (40b) and (41), respectively.

We iterate procedure (i)-(iii) until a convergence is reached. We start the whole calculation from $T=0$ and increase the temperature gradually. We identify $T_{c}$ numerically as the point where we can no longer find a solution of positive $n_{0}$.

When we calculate $\Delta$ for $T<T_{c}$ by using eqs. (36b) and (40b), we observe that the summation of $\tilde{\rho}^{(\mathrm{qp})}(\boldsymbol{k})$ over $\boldsymbol{k}$ diverges. In order to remove it numerically, we have introduced the energy cutoff $\epsilon_{c}$ so that it satisfies $1 \ll \epsilon_{c} \ll 0.3 \delta_{0}^{-2} .14$

For $T>T_{c}$, we put $n_{0}=0, \underline{\Delta}=\underline{0}$ and $\underline{F}=\underline{0}$. The calculation procedures (i)-(iii) are nearly the same except that the chemical potential is now determined by

$$
N=\sum_{\boldsymbol{k} \sigma} \frac{1}{\exp \left[\beta E_{\boldsymbol{k} \sigma}(\mu)\right]-1} .
$$

\section{Numerical Results}

\subsection{Thermodynamic properties}

We have fixed the interaction parameters of eq. (37) as $\delta_{0}=0.0075$ and $c_{2} / c_{0}=0, \pm 0.1$. We have confirmed that the stable state is given by $\boldsymbol{\eta}=[010]^{\mathrm{T}}\left(\boldsymbol{\eta}=[100]^{\mathrm{T}}\right)$ at all temperatures for the antiferromagnetic (ferromagnetic) interaction of $c_{2} / c_{0}=0.1\left(c_{2} / c_{0}=-0.1\right)$. We have found for all these cases that the transition temperature $T_{c}$ is increased over $T_{0}$ by $\left(T_{c}-T_{0}\right) / T_{0} \approx 2 \delta_{0}$, which is of the same order as $\left(T_{c}-T_{0}\right) / T_{0} \simeq 2.33 \delta$ of the single-component case. ${ }^{14,25}$ We have also observed that $T_{c}$ increases monotonically as a function of $c_{2} / c_{0}$.

Figure 1 shows temperature dependence of the condensate density in comparison with the ideal Bose gas result $n_{0} / n=1-\left(T / T_{c}\right)^{3 / 2}$. We observe that $n_{0}$ becomes larger as the interaction is increased. We have also confirmed that $n_{0}$ depends little on $c_{2}$ as long as $\left|c_{2} / c_{0}\right|$ is much smaller than 1.

Figure 2 displays temperature dependence of the specific heat per particle in comparison with the ideal gas result. As seen clearly, a divergent behavior shows up just below $T_{c}$ for the finite interaction. It is quite similar to the behavior of the single-component system.

Figure 3 plots temperature dependence of the chemical potential scaled by $T_{0}$. With finite interaction, we find a small peak with a discontinuity at $T=T_{c}$. This discontinuity is a signal of a first-order transition and also seen



Fig. 1. Temperature dependence of density of particle.

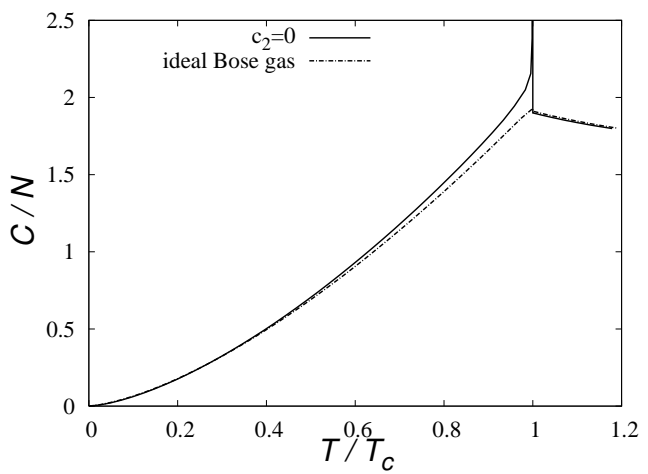

Fig. 2. Temperature dependence of specific heat.

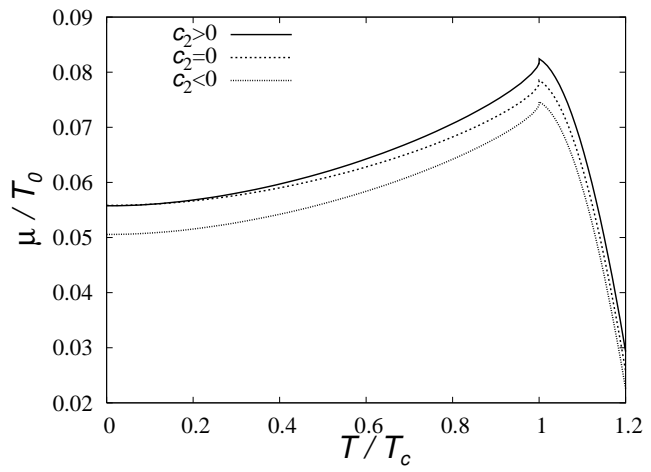

Fig. 3. chemical potential

in the single-component case.

\subsection{Energy dispersion of antiferromagnetic interaction}

We next study the energy dispersion of the antiferromagnetic interaction $\left(c_{2} / c_{0}=0.1\right)$ at $T=0$ and compare our result with the prediction of the Bogoliubov approximation (BA). In BA, the quantities $\mu, \boldsymbol{\eta}$ and $E_{\boldsymbol{k} \sigma}$ can all be obtained analytically as $\mu=c_{0} n_{0}, \boldsymbol{\eta}=[010]^{\mathrm{T}}$, $E_{\boldsymbol{k} 0}=\sqrt{\epsilon_{\boldsymbol{k}}\left(\epsilon_{\boldsymbol{k}}+2 c_{0} n_{0}\right)}$ and $E_{\boldsymbol{k} \pm 1}=\sqrt{\epsilon_{\boldsymbol{k}}\left(\epsilon_{\boldsymbol{k}}+2 c_{2} n_{0}\right)}$ with $\epsilon_{\boldsymbol{k}}=\boldsymbol{k}^{2} / 2 M$; thus, $E_{\boldsymbol{k} \pm 1}$ is degenerate in BA.

Figure 4 shows energy dispersion at $T=0$. The BA predicts that all the three branches have $k$-linear soundwave dispersions in the long wavelength limit and two 


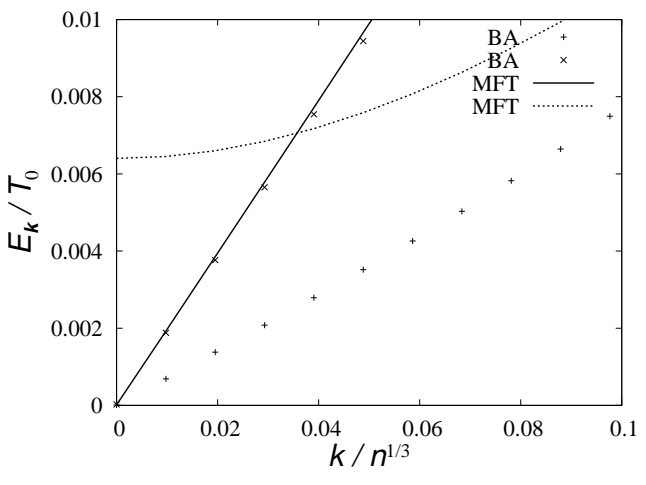

Fig. 4. The energy dispersion of antiferromagnetic interaction at $T=0$

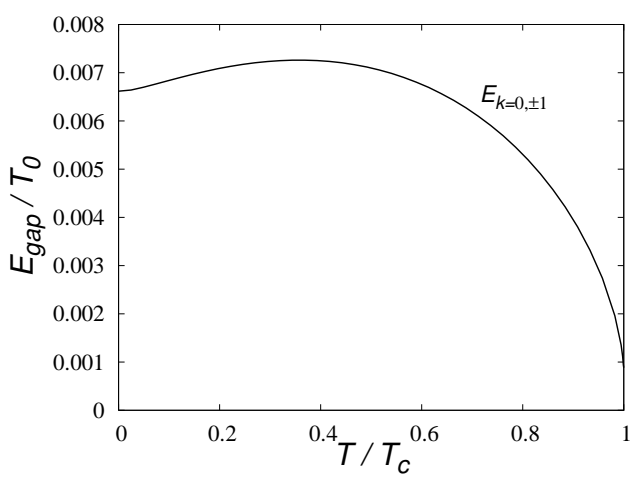

Fig. 5. Temperature dependence of excitation energy gap in antiferro interaction.

of them are degenerate. Our theory also yields a single gapless excitation in agreement with the HugenholtzPines theorem, and its sound velocity $c_{\mathrm{AF}}$ coincides almost completely with the expression $c_{0} n_{0} / M$ of BA for the non-degenerate dispersion, as seen in Fig. 4. As for the other two branches, our mean-field theory predicts degenerate gapful excitations. This gap is brought about by the anomalous pair potential of the quasiparticle field in the BdG equation, which is absent in BA.

The qualitative features of the energy dispersions mentioned above remain invariant up to $T_{c}$. The sound velocity decreases monotonically towards $T_{c}$. In contrast, the energy gap between $E_{\boldsymbol{k}=\mathbf{0}, 0}$ and $E_{\boldsymbol{k}=\mathbf{0}, \pm 1}$ displays a more complicated behavior. Figure 5 shows temperature dependence of the energy gap. The gap has a local maximum as a function of temperature, which may be traced to the temperature dependence of the anomalous pair correlation $\sum_{\boldsymbol{k}} \underline{\tilde{\rho}}(\boldsymbol{k})^{(\mathrm{qp})}$. Indeed, $\sum_{\boldsymbol{k}} \underline{\tilde{\rho}}(\boldsymbol{k})^{(\mathrm{qp})}$ has a local maximum even in the single-component case, which is due to the competition between decreasing $\underline{\Delta}$ and increasing Bose distribution function as $T$ is raised.

We finally comment on the finite energy gap of $E_{\boldsymbol{k}=\mathbf{0}, \pm 1}$ predicted by our mean-field theory in connection with the collective excitations. Gavoret and Nozières ${ }^{28}$ performed a detailed study on the structure of the perturbation expansion for the one-component BEC at $T=0$. They have thereby established that the singleparticle (i.e., Bogoliubov) and collective excitations share common dispersions. Indeed, the statement holds even at any finite temperatures of $T \leq T_{c}$, which stems from the fact that the single-particle propagator appears in the intermediate state of the collective propagator in the condensate; see eq. (2.33) of Szépfalusy and Kondor ${ }^{29}$ and also their discussion in the paragraph below eq. (2.34). However, it should be noted that this theorem only states that the single-particle excitation spectra are also included in the collective excitations, but not vice versa. Thus, it is neither surprising nor contradictory that the collective excitations have extra branches not present in the single-particle excitations.

The theorem may be extended to multi-component systems to tell us that all the single-particle excitation spectra are also present in the collective excitations. It is also worth noting that the Hugenholtz-Pines theorem of the multi-component case only states the existence of a single gapless one-particle excitation spectrum, as shown in Appendix. Thus, the qualitative features of the three branches $E_{\boldsymbol{k} 0}$ and $E_{\boldsymbol{k} \pm 1}$ obtained here satisfy all the exact statements, and it is not contradictory that the collective spin-wave modes are not included in the single-particle excitations.

Recently, Szépfalusy and Szirmai ${ }^{30}$ have extended the consideration of Szépfalusy and Kondor ${ }^{29}$ to the spin1 system. They conclude that there appear additional collective excitations due to the spin-degrees of freedom whose spectra in the long-wavelength limit are also gapless and identical with those of the corresponding singleparticle excitations. This result is clearly in contradiction with the finite energy gap of $E_{\boldsymbol{k}=\mathbf{0}, \pm 1}$ in our mean-field theory. We suspect that the conclusion of Szépfalusy and Szirmai $^{30}$ on the existence of the spin-wave mode in the single-particle spectra is due to an inappropriate assumption on the poles and zeros of the single-particle Green's function expressed with the same denominator as the collective propagators. Note also that they only confirm their general theory within the Bogoliubov approximation and the random-phase approximation of retaining only the Hartree term where the anomalous pair potential due to quasiparticles was neglected completely.

\subsection{Energy dispersion of ferromagnetic interaction}

We move on to study the energy dispersion of the ferromagnetic interaction $\left(c_{2} / c_{0}=-0.1\right)$. In this case, BA near $T=0$ predicts $\mu=\left(c_{0}+c_{2}\right) n_{0}, \boldsymbol{\eta}=[100]^{\mathrm{T}}, E_{\boldsymbol{k}+1}=$ $\sqrt{\epsilon_{\boldsymbol{k}}\left[\epsilon_{\boldsymbol{k}}+2\left(c_{0}+c_{2}\right) n_{0}\right]}, E_{\boldsymbol{k} 0}=\epsilon_{\boldsymbol{k}}$ and $E_{\boldsymbol{k}-1}=\epsilon_{\boldsymbol{k}}-2 c_{2} n_{0}$.

Figure 6 shows the energy dispersions of $E_{\boldsymbol{k}+1}$ and $E_{\boldsymbol{k} 0}$ at $T=0$. According to $\mathrm{BA}$, the two branches are both gapless and $E_{\boldsymbol{k}+1}\left(E_{\boldsymbol{k} 0}\right)$ has a linear (quadratic) dispersion in the long wavelength limit. However, our mean-field theory predicts a gapless excitation only for $E_{\boldsymbol{k}+1}$, and the other branch $E_{\boldsymbol{k} 0}$ acquires an energy gap. The existence of a gapless excitation $E_{\boldsymbol{k}+1}$ agrees with the Hugenholtz-Pines theorem, and its sound velocity $c_{\mathrm{FE}}$ coincides almost completely with the expression $\left(c_{0}+c_{2}\right) n_{0} / M$ of BA. On the other hand, a finite gap in $E_{k 0}$ is brought about by the anomalous pair potential of the quasiparticle field.

We have confirmed that the vector $\boldsymbol{\eta}$ remains invariant at finite temperatures. Also, the qualitative features of 


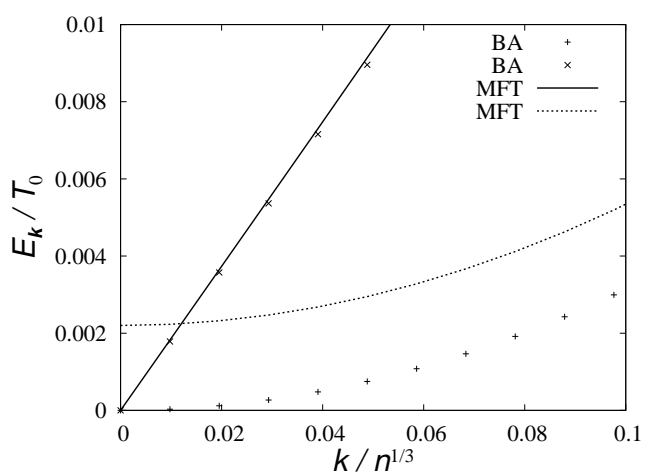

Fig. 6. Energy dispersion of ferromagnetic interaction at $T=0$

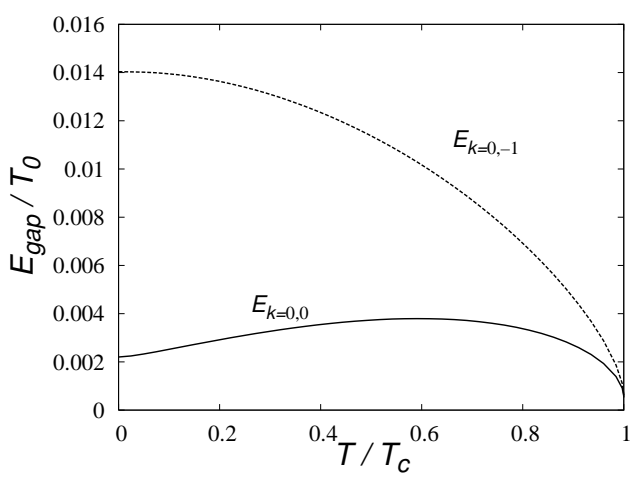

Fig. 7. Temperature dependence of excitation energy gap in ferro interaction.

the dispersions do not change up to $T=T_{c}$. The sound velocity decreases monotonically towards $T_{c}$. Figure 7 shows the energy gaps of $E_{\boldsymbol{k} 0}$ and $E_{\boldsymbol{k}-1}$ at $k=0$ as a function of temperature. The gap of $E_{\boldsymbol{k} 0}$ has a maximum at finite temperature, which has the same origin as in the antiferromagnetic case.

\section{Summary}

We have extended a conserving gapless mean-field theory of single-component Bose-Einstein condensates to a three-component system. The equations to determine the equilibrium are given by eqs. (24) and (25) with eqs. (21), (22) and (29), which are applicable to nonuniform systems such as the trapped BEC with and without vortices. They are compatible with the Hugenholtz-Pines theorem and may be extended easily to general multi-component systems. Another advantage of our theory is that various conservation laws are automatically obeyed when applied to dynamical phenomena. Thus, the static and dynamical properties of BEC may be treated systematically within a single theoretical framework.

We have applied the mean-field theory to a uniform gas with constant density. A qualitative difference is found in the quasiparticle dispersions between our theory and the Bogoliubov theory near $T=0$. The Bogoliubov approximation predicts that there are three (two) gapless excitations for the antiferromagnetic (ferromagnetic) interaction. On the other hand, our theory yields only a single gapless branch with a sound-wave dispersion at low energy, and the others are pushed up to higher energies due to the self-consistent potential of the quasiparticle field. We have presented at the end of $\S 4.2$ a detailed discussion on those single-particle excitations in connection with the collective excitations. The speed of sound obtained here well agrees with the corresponding branch in the Bogoliubov approximation. We have also clarified finite-temperature effects on the quasiparticle dispersions as well as temperature dependences of basic thermodynamic quantities.

\section{Acknowledgement}

This work is supported by the 21st century COE program "Topological Science and Technology," Hokkaido University.

\section{Appendix: Hugenholtz-Pines theorem for spin-1 BEC}

We here generalize the Hugenholtz-Pines theorem ${ }^{12}$ to the spin-1 BEC by closely following the procedure of Popov. ${ }^{31}$ It will be shown that there is at least a single gapless excitation spectrum in the spin-1 BEC (and also in any multi-component BEC).

First of all, we present Feynman rules for the bare perturbation expansion of the spin- 1 condensate as the basis for our proof. They are obtained by slightly modifying the rules of the single-component system. To see this, let us rewrite the interaction of eq. (6) as

$$
\begin{aligned}
\mathcal{H}^{\prime}=\frac{1}{2} \sum_{\nu=0}^{3} \int & \mathrm{d} \boldsymbol{r} \mathrm{d} \boldsymbol{r}^{\prime} \mathcal{U}_{\nu}\left(\boldsymbol{r}-\boldsymbol{r}^{\prime}\right) \\
& \times \mathcal{N}\left[\boldsymbol{\psi}^{\dagger}(\boldsymbol{r}) \underline{A}^{\nu} \boldsymbol{\psi}(\boldsymbol{r}) \boldsymbol{\psi}^{\dagger}\left(\boldsymbol{r}^{\prime}\right) \underline{A}^{\nu} \boldsymbol{\psi}\left(\boldsymbol{r}^{\prime}\right)\right],
\end{aligned}
$$

where $\underline{A}^{\nu}$ is given by eq. (9), and the field operators are defined by

$$
\boldsymbol{\psi}^{\dagger}=\left[\begin{array}{lll}
\psi_{1}^{\dagger} & \psi_{0}^{\dagger} & \psi_{-1}^{\dagger}
\end{array}\right], \quad \boldsymbol{\psi}=\left[\begin{array}{c}
\psi_{1} \\
\psi_{0} \\
\psi_{-1}
\end{array}\right] .
$$

The expression (A.1) enables us to perform the perturbation expansion of the spin-1 system in terms of the Feynman diagrams of the single-component system, i.e., no new diagrams are necessary. To be more specific, we first consider the normal system without the condensation. We then notice that: (i) the pairs $\boldsymbol{\psi}^{\dagger}(\boldsymbol{r}) \underline{A}^{\nu} \boldsymbol{\psi}(\boldsymbol{r})$ and $\boldsymbol{\psi}^{\dagger}\left(\boldsymbol{r}^{\prime}\right) \underline{A}^{\nu} \boldsymbol{\psi}\left(\boldsymbol{r}^{\prime}\right)$ can be moved around anywhere within the $\mathcal{N}$ and/or $T_{\tau}$ operators in the perturbation expansion; and (ii) a contraction of $\boldsymbol{\psi}(i)\left(i \equiv \boldsymbol{r}_{i} \tau_{i}\right)$ with $\boldsymbol{\psi}^{\dagger}(j)$ automatically yields the $3 \times 3$ matrix $\left\langle T_{\tau} \boldsymbol{\psi}(i) \boldsymbol{\psi}^{\dagger}(j)\right\rangle_{0}=-\underline{G}_{0}(i, j)$, where the subscript 0 denotes the average with respect to the non-interacting Hamiltonian. Also, the final contraction within a closed particle loop can be transformed as $\left\langle T_{\tau} \boldsymbol{\psi}^{\dagger}(i) \underline{\mathcal{M}}(i, j) \boldsymbol{\psi}(j)\right\rangle_{0}=\operatorname{Tr} \underline{\mathcal{M}}(i, j)\left\langle T_{\tau} \boldsymbol{\psi}(j) \boldsymbol{\psi}^{\dagger}(i)\right\rangle_{0}$ with $\underline{\mathcal{M}}(i, j)$ denoting some matrix product of contractions. We now realize that the expansion can be performed in terms of the Feynman diagrams of the single-component system with the following modifications: (i) Associate 
$\underline{G}_{0}(i, j)$ for the particle line from $j$ to $i$ and the ma$\operatorname{trix} \underline{A}^{\nu}$ for each vertex, and (ii) take Tr for every closed particle line and perform summations over $\nu$.

We next consider the condensed system and adopt the shift transformation (10) with the self-consistency condition:

$$
\langle\phi(r)\rangle=\mathbf{0} .
$$

Let us substitute eq. (10) into eq. (1) and carry out the perturbation expansion by choosing

$$
\mathcal{H}_{0}=\int \mathrm{d} \boldsymbol{r} \phi^{\dagger}(\boldsymbol{r})\left(H_{0}-\mu\right) \boldsymbol{\phi}(\boldsymbol{r})
$$

as the free field. Once again, we only need the Feynman diagrams of the single-component system, and the Feynman rules for the scalar quantities $G_{0}, \Psi$ and $\Psi^{*}$ are modified as follows: (i) $G_{0}(i, j) \rightarrow \underline{G}_{0}(i, j), \Psi(\boldsymbol{r}) \rightarrow \Psi(\boldsymbol{r})$, and $\Psi^{*}(\boldsymbol{r}) \rightarrow \boldsymbol{\Psi}^{\dagger}(\boldsymbol{r})$; (ii) $\underline{A}^{\nu}$ for the vertex; (iii) take $\mathrm{Tr}$ for every closed particle line and perform summations over $\nu$.

Now, we can directly extend the consideration of Popov $^{31}$ on the general structure of the perturbation expansion to the spin-1 system. We thereby conclude that eq. $(\mathrm{A} \cdot 3)$ is equivalent to the extremal property:

$$
\frac{\delta \Omega}{\delta \boldsymbol{\Psi}^{*}(\boldsymbol{r})}=\mathbf{0} .
$$

It is also straightforward to prove the following relation:

$$
\begin{array}{r}
\frac{\delta^{2} \Omega}{\delta \Psi_{\sigma^{\prime}}\left(\boldsymbol{r}^{\prime}\right) \delta \Psi_{\sigma}^{*}(\boldsymbol{r})}=\Sigma_{\sigma \sigma^{\prime}}\left(\boldsymbol{r}, \boldsymbol{r}^{\prime} ; \omega_{n}=0\right)-\mu \delta_{\sigma \sigma^{\prime}} \delta\left(\boldsymbol{r}-\boldsymbol{r}^{\prime}\right), \\
\frac{\delta^{2} \Omega}{\delta \Psi_{\sigma^{\prime}}^{*}\left(\boldsymbol{r}^{\prime}\right) \delta \Psi_{\sigma}^{*}(\boldsymbol{r})}=\Delta_{\sigma \sigma^{\prime}}\left(\boldsymbol{r}, \boldsymbol{r}^{\prime} ; \omega_{n}=0\right) .
\end{array}
$$

We finally consider the uniform condensate, write the condensate wave function as eq. (32), and expand $\underline{\Sigma}$ and $\Delta$ as eq. (33). It is then evident that the thermodynamic potential depends on $\boldsymbol{\Psi}$ and $\boldsymbol{\Psi}^{\dagger}$ via the product $n_{0} \equiv$ $\boldsymbol{\Psi}^{\dagger} \boldsymbol{\Psi}$. Hence we can write eqs. (A.5) and (A.6) for the uniform case as

$$
\frac{\partial \Omega}{\partial \Psi_{\sigma}^{*}}=\Psi_{\sigma} \frac{\partial \Omega}{\partial n_{0}}
$$

and

$$
\frac{\partial^{2} \Omega}{\partial \Psi_{\sigma^{\prime}} \partial \Psi_{\sigma}^{*}}=\delta_{\sigma \sigma^{\prime}} \frac{\partial \Omega}{\partial n_{0}}+\Psi_{\sigma^{\prime}}^{*} \Psi_{\sigma} \frac{\partial^{2} \Omega}{\partial n_{0}^{2}}
$$

$$
\frac{\partial^{2} \Omega}{\partial \Psi_{\sigma^{\prime}}^{*} \partial \Psi_{\sigma}^{*}}=\Psi_{\sigma^{\prime}} \Psi_{\sigma} \frac{\partial^{2} \Omega}{\partial n_{0}^{2}},
$$

respectively. Using eqs. (A.5)-(A.8), we arrive at the generalized Hugenholtz-Pines theorem:

$$
\begin{aligned}
& \sum_{\sigma^{\prime}}\left[\Sigma_{\sigma \sigma^{\prime}}\left(\boldsymbol{k}=\mathbf{0}, \omega_{n}=0\right) \Psi_{\sigma^{\prime}}-\Delta_{\sigma \sigma^{\prime}}\left(\boldsymbol{k}=\mathbf{0}, \omega_{n}=0\right) \Psi_{\sigma^{\prime}}^{*}\right] \\
& =\mu \Psi_{\sigma} .
\end{aligned}
$$

It tells us that there is at least a single gapless oneparticle excitation in the spin-1 BEC; see the discussion below eq. (39) on this point. It is also straightforward to extend the consideration to any multi-component system, which leads to the same conclusion.

1) D. M. Stamper-Kurn, et. al: Phys. Rev. Lett. 80 (1998) 2027.

2) A. Görlitz, et. al: Phys. Rev. Lett. 90 (2003) 090401.

3) M. D. Barrett, et. al: Phys. Rev. Lett. 87 (2001) 010404.

4) M. Erhard, et. al: Phys. Rev. A. 70 (2004) 031602.

5) M. -S. Chang, et. al: Phys. Rev. Lett. 92 (2004) 140403.

6) H. Schmaljohann, et. al: Phys. Rev. Lett. 92 (2004) 040402.

7) N. N. Bogoliubov: J. Phys. (Moscow) 11 (1947) 23.

8) A. L. Fetter: Ann. Phys. (N.Y.) 70 (1972) 67.

9) E. P. Gross; Nuovo Cimento 20 (1961) 454.

10) E. P. Gross; J. Math. Phys. 4 (1963) 195.

11) L. P. Pitaevskii; Sov. Phys.-JETP 13 (1961) 451.

12) N. M. Hugenholtz and D. Pines: Phys. Rev. 116 (1959) 489.

13) N. Shohno: Progr. Theoret. Phys. (Kyoto) 31 (1964) 553; 32 (1964) 370.

14) T. Kita: J. Phys. Soc. Jpn. 75 (2006) 044603.

15) P. C. Hohenberg and P. C. Martin: Ann. Phys. (N.Y.) 34 (1965) 291.

16) T. Ohmi, K. Machida: J. Phys. Soc. Jpn. 67 (1998) 1822.

17) T. -L. Ho: Phys. Rev. Lett. 81 (1998) 742.

18) C. K. Law et. al: Phys. Rev. Lett 81 (1998) 5257.

19) H. Pu et. al: Phys. Rev. A 60 (1999) 1463.

20) W. Zhang et. al: Phys. Rev. Lett 95 (2005) 180403.

21) J. Mur-Petit et. al: Phys. Rev. A 73 (2006) 013629.

22) W.-J Huang and S.-C. Gou: Phys. Rev. A 59 (1999) 4608.

23) T. Isoshima et. al: J. Phys. Soc. Jpn. 69 (2000) 3864.

24) W. Zhang et. al: Phys. Rev. A 70 (2004) 043611.

25) T. Kita: J. Phys. Soc. Jpn. 74 (2005) 1891; T. Kita: J. Phys. Soc. Jpn. 74 (2005) 3397.

$26)$ A. L. Fetter, J. D. Walecka, Quantum theory of many particle systems, Dover Pubns (2003)

27) L. M. Luttinger and L. C. Ward: Phys. Rev. 118 (1960) 1417.

28) J. Gavoret and P. Nozières: Ann. Phys. (NY) 28 (1964) 349.

29) P. Szépfalusy and I. Kondor: Ann. Phys. (NY) 82 (1974) 1.

30) P. Szépfalusy and G. Szirmai: Phys. Rev. A 65 (2002) 043602.

31) N. V. Popov: Functional Integrals and Collective Excitations (Cambridge Univ. Press, Cambridge, 1987) Sec. 5. 